\title{
Spectral analysis of S5 $1803+784$ in the recent flaring state
}

Joseph Omojola ${ }^{a, b,{ }^{*}}$ and Andrew Chen ${ }^{a}$ on behalf of the Fermi-LAT Collaboration

${ }^{a}$ School of Physics, University of the Witwatersrand,

2050, Johannesburg, South Africa

${ }^{b}$ Department of Physics, Federal University of Lafia, 950101, Lafia, Nigeria

E-mail:1926749@students.wits.ac.za,Andrew.Chen@wits.ac.za

S5 $1803+784$ is a low synchrotron peaked (LSP) BL Lac object. Recent multiwavelength studies show no clear correlation between the synchrotron emission and the $\gamma$-ray emission in this blazar. Unlike most LSP blazars, the spectrum of S5 $1803+784$ is poorly fitted with a single zone synchrotron self-Compton (SSC) leptonic jet model. We utilize a simple single-zone leptonic jet emission model with external Compton (EC) originating from the dusty torus (DT) to explain the emission and produce the best-fit parameters. The spectral analysis of the blazar in the flaring state reported in Astronomers' Telegram (ATel \#13633) is studied using data from Fermi-LAT and NED archival data. We verified that the NED archival data are suitable for the spectral analysis of this blazar both during the flare and in the quiescent state by plotting the available synchrotron simultaneous data of the flare period with the NED archival data. The spectral energy distribution (SED) model of the flare and the quiescent state's best-fit parameters are produced using the JetSeT code. The results constrain the upper limit of the $\gamma$-ray emission region length scale, the jet energetics, and the likely acceleration mechanism of the blazar during the flare. 


\section{Introduction}

Leptonic models comprise a jet emission mechanism in which ultra-relativistic electrons and positrons up-scatter soft photons of synchrotron emission within the jet itself or soft photons external to the jet in an inverse Compton process [1]. The single-zone SSC emission model has successfully explained the spectral energy distribution (SED) and variability of many blazars. Still, others require a different outlook to explain their unique characteristics [2]. The location of the $\gamma$-ray emission site in blazars is not yet ascertained. For example, $\gamma$-rays may be produced in the radio-emitting regions [3] or closer to the central engine. Unlike in the radio, at high energies, the angular resolution is insufficient to determine the precise location of the emission region; therefore, the size of the emission region, acceleration mechanisms, and location must be inferred from flux variations and phenomenological spectral fits. We perform a phenomenological fit of the recent flaring state of this blazar using a single-zone SSC + EC jet leptonic model with the external photons as reprocessed infrared photons from the dusty torus (DT).

We adopt the definition of flare proposed in [4]. A flare is a continuous period associated with a given flux peak, during which the flux exceeds the quiescent value, and the lower limit is attained twice at the beginning and the end of the flare. We use the lambda cold dark matter $(\Lambda \mathrm{CDM})$ cosmology, $H_{0}=71 \mathrm{~km} \mathrm{~s}^{-1} \mathrm{Mpc}^{-1}, \Omega_{\mathrm{m}}=0.27, \Omega_{\Lambda}=0.73$.

\section{S5 1803+784 (4FGL J1800.6+7828)}

Fermi-LAT observed $\gamma$-ray flaring activity from S5 1803+784 (4FGL J1800.6+7828 in Fermi-LAT 4FGL catalog) [5], at a redshift $\mathrm{z}=0.684$. The flaring activity is observed from MJD 58941 - MJD 58958. The highest daily flux ever observed for this source is on 12 April 2020 (MJD 58951) at $(1.5 \pm 0.2) \times 10^{-6}$ photons $\mathrm{cm}^{-2} \mathrm{~s}^{-1}$. The Fermi-LAT log-parabola energy spectrum in the time range MJD 58941 - MJD 58958 with a cut-off at $\sim 13 \mathrm{GeV}$ is shown in Figure 1A, while Figure 1B is the NED archival data and the OVRO data are time-averaged over the flare period. The optical data from Nesci et al. [6] is plotted therein to show that NED archival data can represent the low energy range and could produce a good spectral fit for this blazar in both states. 

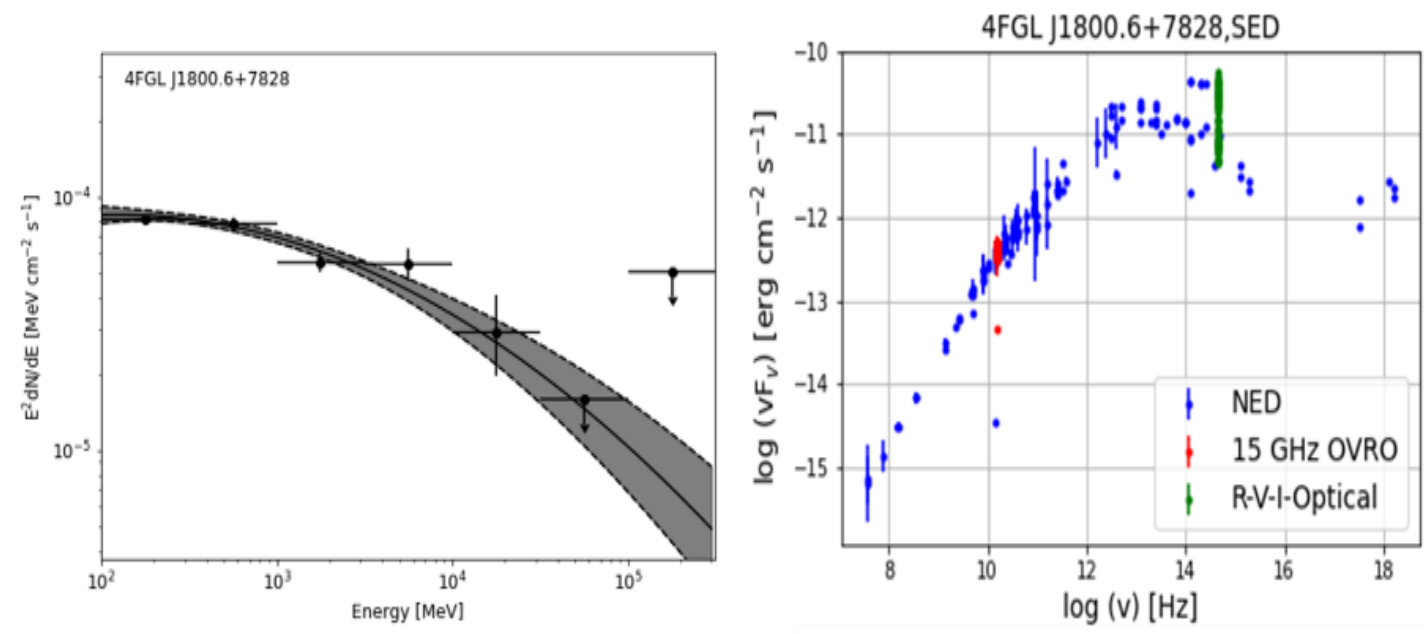

Figure 1: The SED in (A) Fermi-LAT range (B) NED archival data, the simultaneous $15 \mathrm{GHz}$ OVRO data, and optical data from [6] were compared with NED data used in the spectral fit.

\section{Fermi-LAT and NED data analysis}

The reprocessed Pass 8 data sets with P8R3_SOURCE_V2 instrument response functions were used to analyze the data following the standard procedures [7] implemented in the Fermi ScienceTools (ver. 1.2.23) software package using Fermipy (0.20.0) [8]. The statistical significance of the HE $\gamma$-ray signal in the energy range $0.1-300 \mathrm{GeV}$ was determined from the maximum likelihood ratio test statistic (TS) defined in [9]. The SED of the flare announced in ATel \#13633, which peaked on 12 April 2020 (MJD 58951) within ten days (MJD 58948 - MJD 58957) of the flare, was extracted in the energy bins centered on 177.83, 562.34, 1778.28, $5623.41,17782.79,56234.13$, and $177827.94 \mathrm{MeV}$. The flux densities with $\mathrm{TS} \geq 20$ were used to build the SEDs. The high-energy portion of the quiescent state of the SED was obtained from the 4FGL catalog. The archival data of the blazar from the radio up to the X-ray was obtained from the NASA/IPAC Extragalactic Database (NED) at ned.ipac.caltech.edu.

\section{Results and Discussion}

\subsection{Model description, JetSeT code setting, and results}

The electron injection is described by a broken power-law function in the JetSeT code [10] in the framework of the jet leptonic model. The phenomenological spectral fit in JetSeT uses the Minuit minimizer, and the observational data are binned at 0.2 bin width in logarithmic frequency space to obtain a best-fit model for the fixed parameter in Table 1 and the SEDs in Figures 2 and 3. With the Monte Carlo Markov chain (MCMC) sampler embedded in JetSeT, we obtain the best fit of the SED in Table 2, and the phenomenological SED fits in Figures 4 and 5. The lower and 
upper bounds of the uncertainty are the differences between the 16th and 84th percentiles in the probability density function. The parameters are quite well constrained by the data.

Table 1: Fixed parameters in the spectral fit of S5 1803+784.

\begin{tabular}{|l|l|c|c|}
\hline Symbol (unit) & Description & Quiescent & Flare \\
\hline $\mathrm{R}_{\mathrm{H}} \times 10^{18} \mathrm{~cm}$ & $\begin{array}{l}\text { Emitting region position from the black } \\
\text { hole }\end{array}$ & 1.00 & 1.00 \\
\hline $\mathrm{L}_{\mathrm{Disk}} \times 10^{44} \mathrm{erg} / \mathrm{s}$ & Disk luminosity & 2.12 & 2.30 \\
\hline $\mathrm{R}_{\mathrm{DT}} \times 10^{18} \mathrm{~cm}$ & Size of the dusty torus & 1.15 & 1.20 \\
\hline $\mathrm{R}_{\mathrm{BLR}-\mathrm{in}} \times 10^{16} \mathrm{~cm}$ & Inner radius of the BLR & 4.60 & 4.80 \\
\hline $\mathrm{R}_{\mathrm{BLR}-\text { out }} \times 10^{16} \mathrm{~cm}$ & Outer radius of the BLR & 9.20 & 9.60 \\
\hline $\mathrm{T}_{\mathrm{Disk}} \times 10^{4} \mathrm{~K}$ & Disk temperature & 3.02 & 3.02 \\
\hline tau & $\begin{array}{l}\mathrm{BLR} \\
\text { Proportion of external photons } \\
\text { rau }\end{array}$ & 0.1 & 0.1 \\
\hline $\mathrm{T}_{\mathrm{DT}}(\mathrm{K})$ & $\begin{array}{l}\text { Proportion of external photons } \\
\text { re-emitted from the DT }\end{array}$ & 0.1 & 0.1 \\
\hline & Temperature of the dusty torus & 655 & 655 \\
\hline
\end{tabular}

Table 2: Free parameters and best-fit results of S5 1803+784.

\begin{tabular}{|c|l|l|l|}
\hline Symbol (unit) & Description & Quiescent & Flare \\
\hline$\gamma_{\mathrm{b}} \times 10^{3}$ & $\begin{array}{l}\text { Turn-over-energy } \\
\text { Lorentz factor }\end{array}$ & $0.52_{-0.047}^{+0.061}$ & $0.63_{-0.068}^{+0.076}$ \\
\hline $\mathrm{p}$ & $\begin{array}{l}\text { Low energy spectral } \\
\text { slope }\end{array}$ & $-0.82_{-0.12}^{+0.11}$ & $-0.56_{-0.18}^{+0.18}$ \\
\hline $\mathrm{p}_{1}$ & $\begin{array}{l}\text { High energy spectral } \\
\text { slope }\end{array}$ & $3.54_{-0.11}^{+0.13}$ & $3.54_{-0.10}^{+0.11}$ \\
\hline$\gamma_{\min }$ & $\begin{array}{l}\text { Low-energy-cut-off } \\
\text { Lorentz factor }\end{array}$ & $5.97_{-1.00}^{+0.89}$ & $13.66_{-3.65}^{+1.13}$ \\
\hline$\gamma_{\max } \times 10^{5}$ & $\begin{array}{l}\text { High-energy-cut-off } \\
\text { Lorentz factor }\end{array}$ & 1.02 & $1.16_{-0.27}^{+0.28}$ \\
\hline $\mathrm{R}(\mathrm{cm}) \times 10^{16}$ & Emitting region size & $19.79_{-2.20}^{+1.96}$ & $9.66_{-1.31}^{+1.43}$ \\
\hline$\delta$ & Doppler factor & $12.20_{-1.20}^{+1.35}$ & $26.79_{-2.41}^{+2.24}$ \\
\hline $\mathrm{B}(\mathrm{G})$ & Magnetic field & $0.36_{-0.03}^{+0.03}$ & $0.13_{-0.01}^{+0.01}$ \\
\hline $\mathrm{N}\left(\mathrm{cm}^{-3}\right)$ & Emitter density & $3.10_{-0.43}^{+0.46}$ & $8.70_{-1.11}^{+1.34}$ \\
\hline
\end{tabular}

\section{$4.2 \gamma$-ray emission and acceleration processes}

The phenomenological spectral best fit requires external photons from the dusty torus for the $\gamma$-ray emission (Figures 2 and 3). The spectral best fit values of $\delta$ obtained in Table 2 are within the range observed in the VLBI observations of S51803+784 [11]. The quiescent $\delta$ is the value obtained using the eight years catalog average. The ratio of the magnetic energy density and particle energy density in the emitting region is $\left(\mathrm{U}_{\mathrm{B}} / \mathrm{U}_{\mathrm{e}}\right)=6.29 \times 10^{-4} / 4.45 \times 10^{-3}=0.14$ during the flare and $5.15 \times 10^{-3} / 1.39 \times 10^{-3}=3.70$ in the quiescent state of the blazar, implying 
a particle-dominated emitting region during the flare. The above implies a possibility of magnetic dissipation in the emitting region. The jet is also magnetically dominated during the quiescent state but particle dominated during the flaring state. This scenario favors an enhanced particle acceleration through magnetic reconnection during the flare [12].

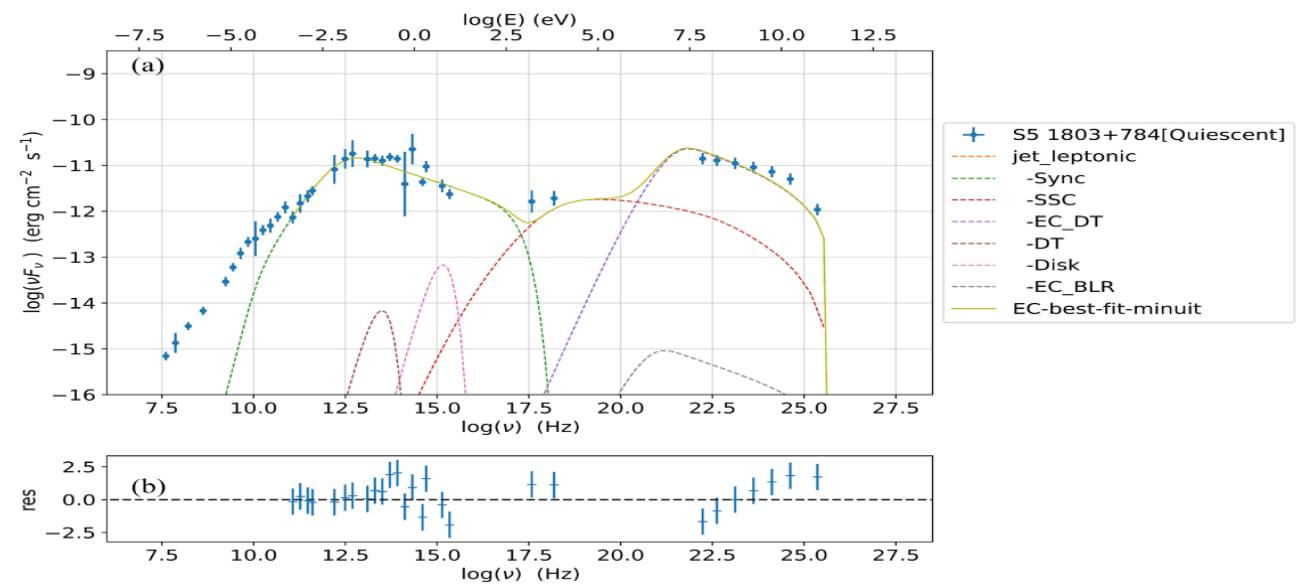

Figure 2: (a) S5 1803+784 broadband best-fit SED (Quiescent state); (b) the lower subpanel shows the residual of the fit.
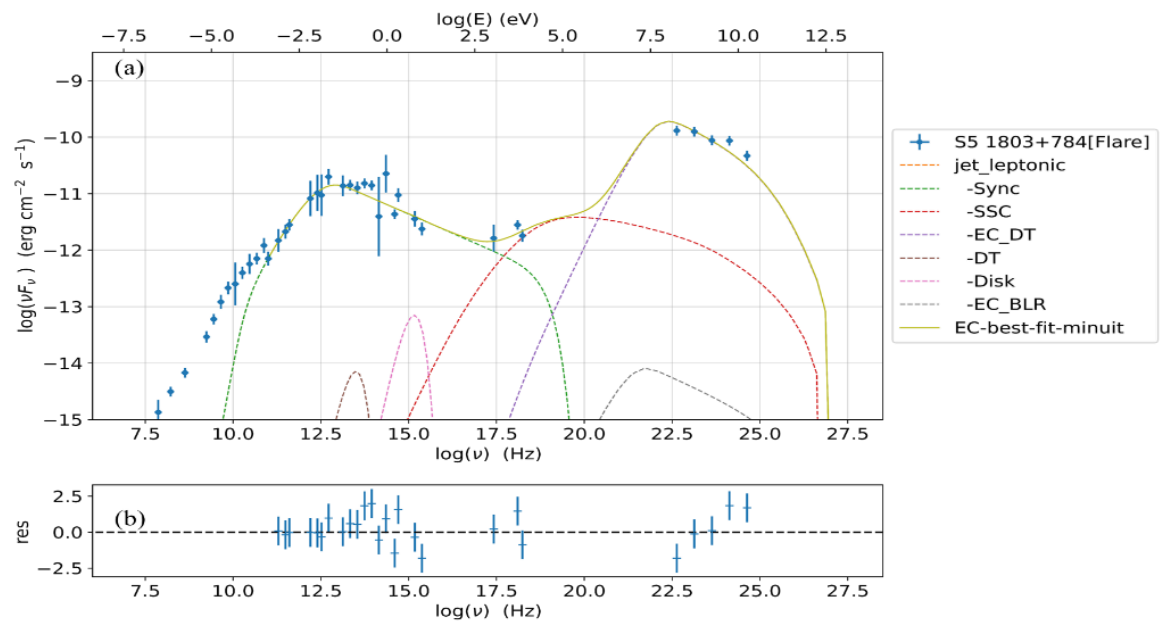

Figure 3: (a) S5 1803+784 broadband best-fit SED (Flaring state); (b) the lower subpanel shows the residual of the fit.

\section{Conclusion}

The jet energetics and the phenomenological spectral best fit for both the quiescent and flaring state of the blazar are reported in Figures 2, 3, 4, and 5, whereby the synchrotron emission of the extended jet is not included. The lack of clear cross-correlation between the radio and the $\gamma$ ray bands for this source [6] could be explained by the fact that the $\gamma$-ray emission is due to upscattered soft infrared photons originating from the dusty torus. This blazar may be a masquerading BL Lac [13] since the observational evidence of a dusty torus is still lacking for BL Lacs. 
The flare that peaks on MJD 58951 from this source presents signatures and features consistent with magnetic dissipation through magnetic reconnection as a process enhancing particle acceleration.

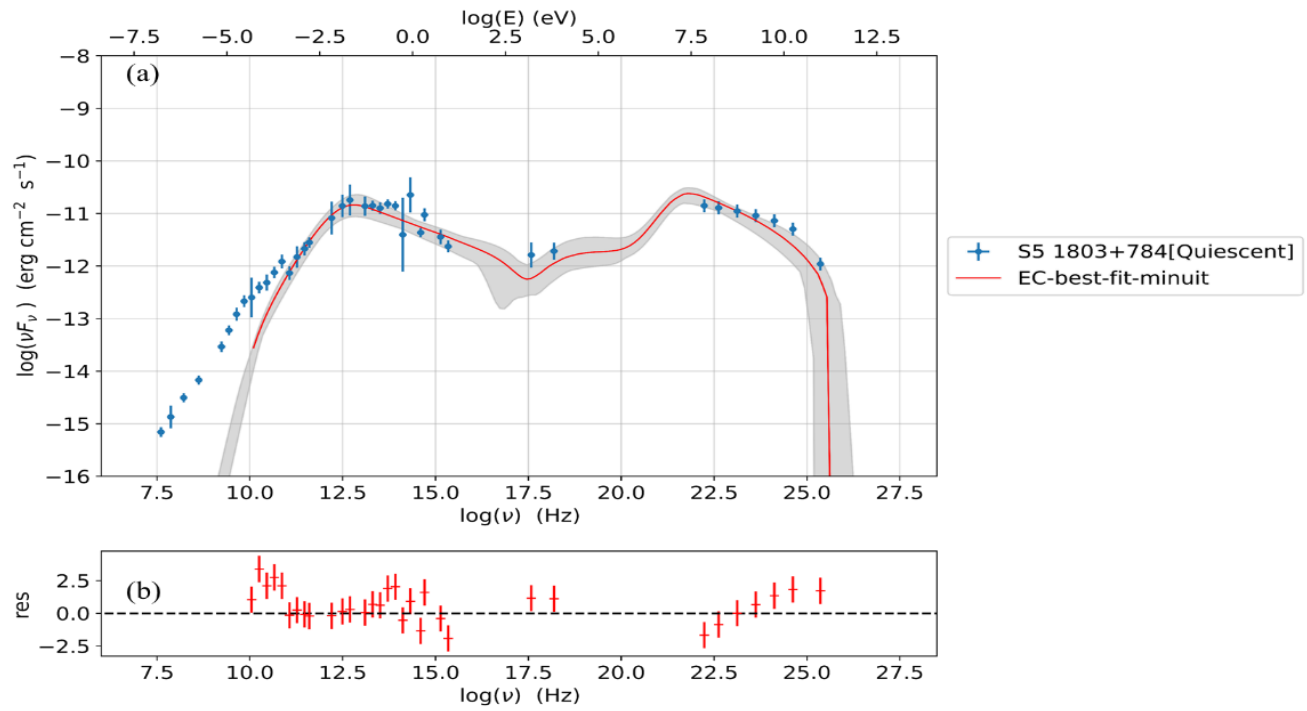

Figure 4: (a) Phenomenological spectral best-fit of S5 1803+784 (Quiescent state); (b) the lower subpanel shows the residual of the fit.
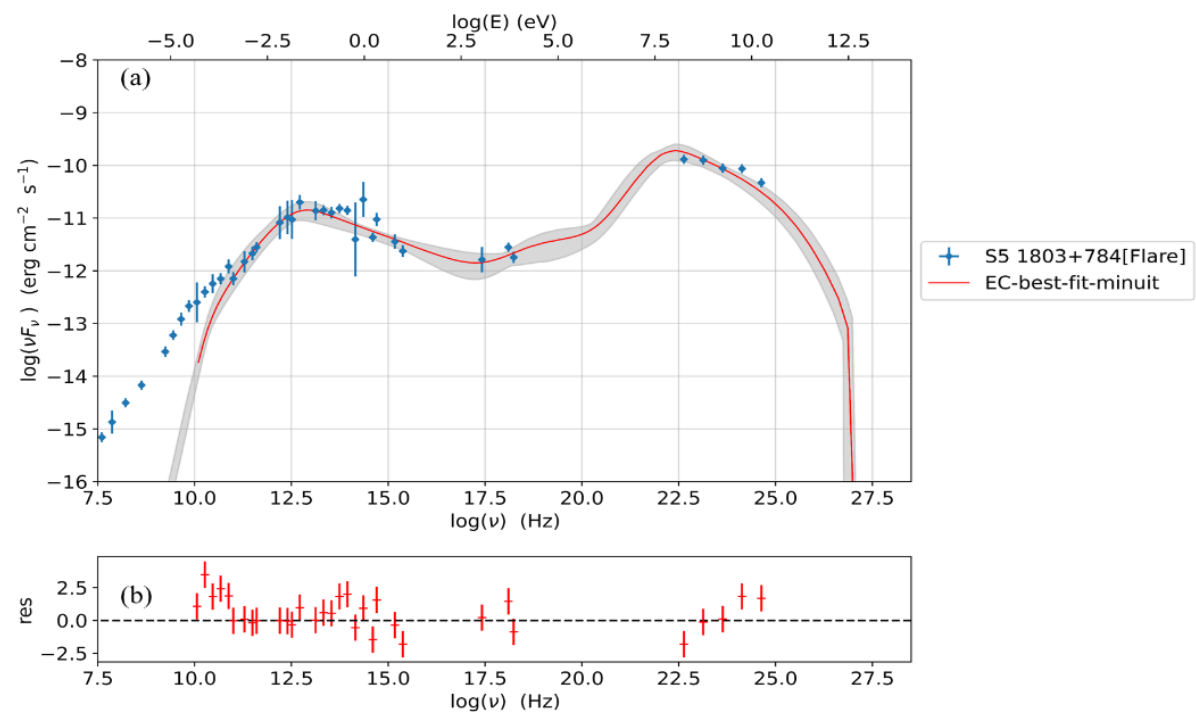

Figure 5: (a) Phenomenological spectral best-fit of S5 1803+784 (Flaring state); (b) the lower subpanel shows the residual of the fit.

Acknowledgments: The Fermi-LAT Collaboration acknowledges support for LAT development, operation, and data analysis from NASA and DOE (United States), CEA/Irfu and IN2P3/CNRS (France), ASI and INFN (Italy), MEXT, KEK, and JAXA (Japan), and the K.A. Wallenberg Foundation, the Swedish Research Council and the National Space Board (Sweden). Science analysis support in the operations phase from INAF (Italy) and CNES (France) is also gratefully acknowledged. This work was performed in part under DOE Contract DE-AC02-76SF00515. Also, Andrea Tramacere for the JetSeT code used in the SED modeling. We also use data from the OVRO 40-m monitoring program (Richards, J. L. et al., 2011, ApJS, 194, 29), which is supported in part by NASA grants NNX08AW31G, NNX11A043G and NNX14AQ89G and NSF grants AST - 0808050 and AST - 1109911. 


\section{References}

[1] Böttcher M 2019 Galaxies 7(1) 20

[2] Hervet O, Boisson C and Sol H 2015 A\&A 578 A69

[3] Jorstad S.G et al. 2005 AJ 130 1418-1465

[4] Nalewajko K 2013 MNRAS 430 1324-1333

[5] Abdollahi et al. 2020 ApJS 2471

[6] Nesci R et al. 2021 MNRAS 502 6177-6187.

[7] Abdo A.A, Ackermann M, Ajello M et al. 2010 ApJ 722:520-542

[8] Wood M, Caputo R, Charles E et al. $201735^{\text {th }}$ ICRC2017 301824

[9] Mattox J.R, Bertsch D L, Chiang J et al. 1996 ApJ 461 396-407

[10] Tramacere A. JetSeT: Numerical modeling and SED fitting tool for relativistic jets. Astrophysics Source Code Library. 2020 Sep 1;ascl:2009.001.

[11] Hovatta T et al. 2009 A\&A $494527-537$

[12] Sironi L, Petropoulou M and Giannios D 2015 MNRAS 450 183-191

[13] Giommi P, Padovani P and Polenta G 2013 MNRAS 431 1914-22 